\title{
Macrolide Therapy and Alveolar Hemorrhage Secondary to Granulomatosis with Polyangiitis
}

\author{
George H B Greenhall ${ }^{1}$, Rama Vancheeswaran ${ }^{2}$, Aine Burns ${ }^{1}$, Paul Bass $^{1,3}$ and Alan D Salama ${ }^{{ }^{*}}$ \\ ${ }^{1}$ UCL Centre for Nephrology, Royal Free Hospital, London \\ ${ }^{2}$ Department of Respiratory Medicine, Barnet General Hospital, London \\ ${ }^{3}$ Department of Cellular Pathology, Royal Free Hospital, London
}

*Corresponding author: Salama AD, UCL Centre for Nephrology, Royal Free Hospital, Rowland Hill Street, London, United Kingdom, Tel: +44 207 794 0500 ; Fax. +44 207317 8591; Email: a.salama@ucl.ac.uk

Received date: Sep 06, 2015; Accepted date: Oct 06, 2015; Published date: Oct 09, 2015

Copyright: (c) 2015 Greenhall G, et al. This is an open-access article distributed under the terms of the Creative Commons Attribution License, which permits unrestricted use, distribution, and reproduction in any medium, provided the original author and source are credited.

\begin{abstract}
We report the case of a 64-year-old woman with severe alveolar haemorrhage secondary to granulomatosis with polyangiitis (GPA), who was unknowingly treated with only penicillin and macrolide antibiotics, resulting in successful initial disease remission. The immunomodulatory effects of the macrolide antibiotic used could underlie this novel observation, which may shed new light on the pathogenesis and potential treatment of anti-neutrophil cytoplasm antibody (ANCA) associated vasculitis. The role of macrolides as an adjunct to conventional therapy in cases of AAV with respiratory involvement may be worthy of further investigation.
\end{abstract}

Keywords: Vasculitis; Anti-bacterial agents; Lung disease; Renal disease

\section{Introduction}

Alveolar haemorrhage is one of the most serious manifestations of GPA, which can be rapidly fatal if untreated. Standard immunosuppressive treatment for GPA carries significant toxicity, and on-going research seeks better-tolerated, efficacious regimens. We present a case whose clinical course may be informative with regards pathogenesis and management of AAV.

\section{Case Report}

A 64-year-old woman presented with a four-week history of haemoptysis, fever and malaise. She had no previous medical history, aside from high-frequency hearing loss for several years. Examination revealed temperature of $38.3^{\circ} \mathrm{C}$, bronchial breathing at the right lung base, and hypoxia $\left(\mathrm{PaO}_{2} 8.5 \mathrm{kPa}\right.$ on room air).

Laboratory studies demonstrated anaemia (haemoglobin $64 \mathrm{~g} / \mathrm{L}$ ), leucocytosis $\left(13.9 \times 10^{3}\right.$ cells $/ \mu \mathrm{L}$ ), normal renal function (creatinine 76 $\mu \mathrm{mol} / \mathrm{L})$, and elevated C-reactive protein $(456 \mathrm{mg} / \mathrm{L} ; \mathrm{NR}<10 \mathrm{mg} / \mathrm{L})$. Chest $\mathrm{x}$-ray revealed extensive consolidation affecting the right middle and upper lobes (Figure 1A). Fibreoptic bronchoscopy found purulent secretions and fresh blood in the right lung. Culture of sputum and broncho-alveolar lavage yielded no bacterial organisms, and staining for acid-fast bacilli was negative.

Treatment with intravenous piperacillin-tazobactam and clarithromycin was commenced, and two units of red cells were transfused. She received no inhaled, oral or intravenous corticosteroids. Her condition improved rapidly, and she was discharged after eight days. A repeat chest $\mathrm{x}$-ray at follow-up two months later showed complete resolution of the abnormalities (Figure 1B).

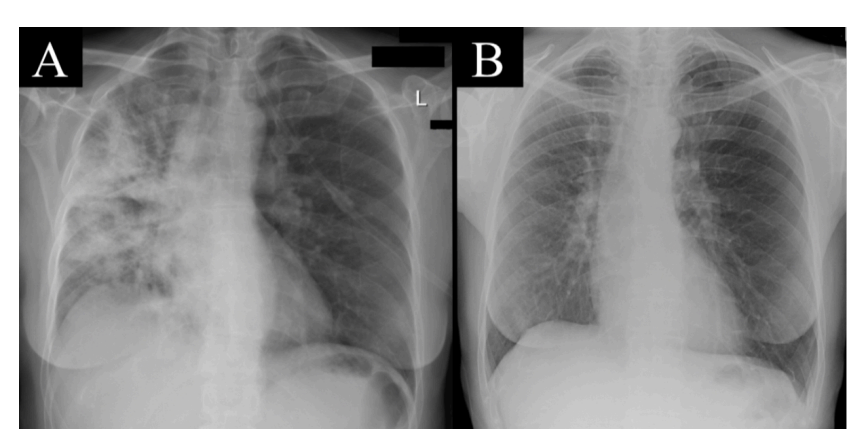

Figure 1: a) Chest radiograph at presentation demonstrating significant airspace shadowing in the right middle and upper lobes. b) Chest radiograph after two months and following antibiotic treatment showing complete resolution of the previous findings.

On retrospective review, serum taken at presentation demonstrated positive immunofluorescence staining for ANCA in a cytoplasmic pattern, with antibodies to proteinase-3 (PR3) at a titre of $143 \mathrm{IU} / \mathrm{mL}$ $(\mathrm{NR}<3 \mathrm{IU} / \mathrm{ml})$. Anti-glomerular basement membrane antibodies were negative. Urine analysis detected microscopic haematuria $(3+$ red blood cells on dipstick), and no proteinuria. Culture of nasal swabs yielded no organisms.

For ten months after her initial presentation, the patient remained entirely well, with normal blood pressure and renal function, and no respiratory symptoms. Microscopic haematuria persisted, and her PR3-ANCA titre remained elevated $(120 \mathrm{IU} / \mathrm{mL})$. She then developed haemoptysis and malaise, acute kidney injury (creatinine $188 \mu \mathrm{mol} / \mathrm{L}$ ), anaemia (haemoglobin $88 \mathrm{~g} / \mathrm{L}$ ) and an increase in PR3-ANCA titre $(143 \mathrm{IU} / \mathrm{mL})$. Chest $\mathrm{x}$-ray revealed consolidation in the left lower zone, and her carbon monoxide transfer factor was elevated (110\%). A renal biopsy (Figure 2) showed pauci-immune focal segmental necrotizing 
crescentic glomerulonephritis. She was treated with intravenous glucocorticoids, cyclophosphamide and rituximab, which resulted in a rapid clinical response. Her disease has since followed a relapsing course, and she is now receiving regular rituximab to maintain remission.

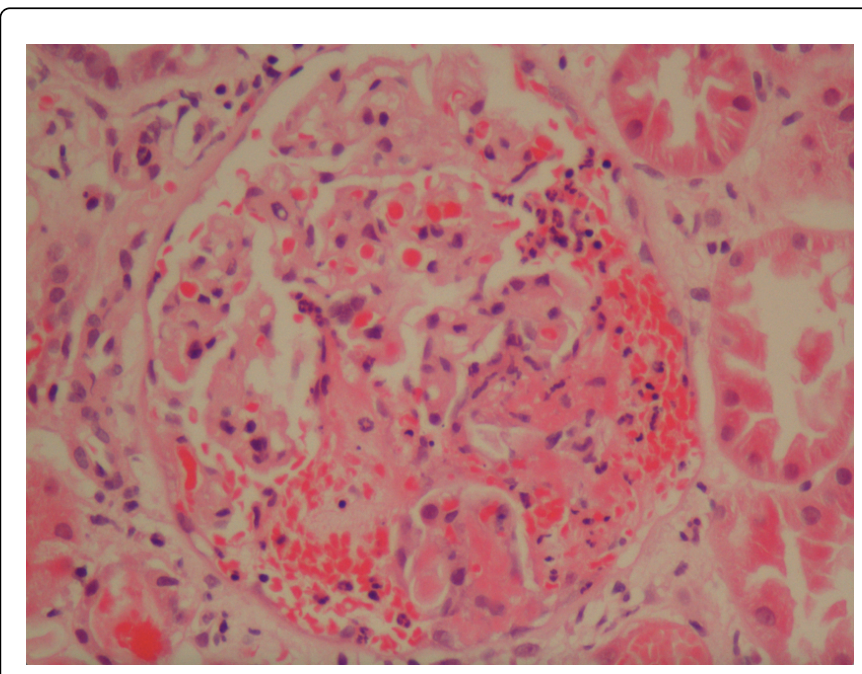

Figure 2: Photomicrograph of section of renal biopsy, showing focal segmental necrotizing crescentic glomerulonephritis (Haematoxylin and Eosin, x40 magnification).

\section{Discussion}

The initial presentation of the case described is clearly consistent with a diagnosis of severe alveolar haemorrhage secondary to pulmonary GPA, and had the ANCA result been available early on during the course of her disease, she would undoubtedly have received immunosuppressive treatment for ANCA associated vasculitis with pulmonary haemorrhage. However, due to the delay in reaching this diagnosis, standard immunosuppressive treatment was not administered. Interestingly, following treatment with only beta-lactam and macrolide antibiotics she had complete resolution of disease, and remained well for ten months. We are unaware of any similar observations in the published literature. This observation could be explained in two different ways: first, that pulmonary vasculitis was provoked by an unidentified bacterial pathogen, the eradication of which eliminated the stimulus for persistent autoimmune damage; second, that the disease was not provoked by infection and remission resulted from an immunomodulatory effect of the antibiotics used. Below, we discuss the evidence for each of these theories.

Bacterial infection is widely considered as a potential trigger of GPA, a theory which was advanced when the disease was first described. Mechanisms through which infection may result in a loss of tolerance and induction of autoimmunity include neutrophil "priming" by inflammatory cytokines, molecular mimicry of self- and pathogenderived peptides, stimulation of auto-reactive lymphocytes by bacterial super-antigen, and modulation of toll-like receptor (TLR) signalling [1]. Clinically, nasal and bronchial carriage of Staphylococcus aureus are associated with disease activity [2,3] and there are also reports of AAV in association with other bacterial [4] and viral [5] infections. In terms of antimicrobial therapy, it has been shown that eradication of nasal $S$. aureus reduces the rate of relapse [6], however, the role of adjunctive antibiotics in induction therapy is unproven, beyond pneumocystis prophylaxis with cyclophosphamide. The only trial directly examining this question found that benefit from cotrimoxazole was limited to patients with disease confined to the respiratory tract, and no constitutional symptoms; notably, one third of the patients in this study had previously received immunosuppression [7]. To our knowledge there are no reports of AAV in any organ system responding to treatment with beta-lactam or macrolide antibiotics.

Macrolide antibiotics share a common structure based around a macrocyclic lactone ring. As well as being effective against many grampositive and some gram-negative bacteria, their immunomodulatory properties are well-described [8]. Various agents from this class of drug are used in the treatment of chronic inflammatory respiratory diseases [9], with anti-inflammatory effects that are likely independent of their antimicrobial action [10]. Experimental and clinical studies show that macrolides cause increased leucocyte apoptosis, reduced cytokine expression, and impaired T-cell proliferation and signalling [11]. Administration of oral clarithromycin to patients with bronchiectasis has been shown to cause local and systemic modulation of the Th17 axis [12], which has also been implicated in the pathogenesis of AAV [13]. Tacrolimus and rapamycin (sirolimus) are non-antimicrobial macrolides widely used in transplantation medicine. Though there is no evidence for the use of these agents in AAV, recent trials support the use of tacrolimus as an adjunct in the treatment of lupus nephritis [14]. Experimental evidence also suggests a role for rapamycin in the treatment of rapidly progressive glomerulonephritis [15]. We have found no published reports on the use of traditional (antimicrobial) macrolides in experimental or clinical vasculitis.

It is certainly possible that the response observed in our patient was due to the eradication of a bacterial stimulus. However, it is perhaps unusual to culture no organisms in such a severe case of pneumonia. In addition, the antecedent history of hearing loss and sub-acute onset of symptoms suggest that a vasculitic process was already established. Ultimately, it is impossible to prove which of the two theories outlined above is correct, and it may have been a combination of the two factors; however, our case is unusual and striking, and potentially supports the immunomodulatory properties of macrolide antibiotics. We believe that other physicians may have noticed similar clinical patterns but not reported them. A more widespread finding could support a formal study of adjunctive macrolide therapy in AAV induction therapy.

In summary, we present a case of relapsing GPA, initially manifesting with severe alveolar haemorrhage, in whom prolonged remission was unknowingly achieved with antibiotic therapy alone. The immunomodulatory effect of clarithromycin is likely to have contributed to this clinical finding. Whilst we of course do not advocate using macrolide antibiotics alone for the treatment of GPA, their adjunctive use may be worthy of further investigation.

\section{References}

1. Tadema H, Heeringa P, Kallenberg CGM (2011) Bacterial infections in Wegener's granulomatosis: mechanisms potentially involved in autoimmune pathogenesis. Curr Opin Rheumatol 23: 366-371.

2. Stegeman CA, Tervaert JW, Sluiter WJ, Manson WL, Jong PE de, et al. (1994) Association of chronic nasal carriage of Staphylococcus aureus and higher relapse rates in Wegener granulomatosis. Ann Intern Med 120: $12-17$. 
Citation: Greenhall G, Vancheeswaran R, Burns A, Bass P, Salama AD (2015) Macrolide Therapy and Alveolar Hemorrhage Secondary to Granulomatosis with Polyangiitis. J Vasc 1: 1000101. doi:10.4172/2471-9544.1000101

Page 3 of 3

3. Richter AG, Stockley RA, Harper L, Thickett DR (2009) Pulmonary infection in Wegener granulomatosis and idiopathic pulmonary fibrosis. Thorax 64: 692-697.

4. Zycinska K, Wardyn KA, Zycinski Z, Smolarczyk R (2008) Correlation between Helicobacter pylori infection and pulmonary Wegener's granulomacytosis activity. J Physiol Pharmacol 6: 845-851.

5. Yamaguchi M, Yoshioka T, Yamakawa T, Maeda M, Shimizu H, et al. (2014) Anti-neutrophil cytoplasmic antibody-associated vasculitis associated with infectious mononucleosis due to primary Epstein-Barr virus infection: report of three cases. Clin Kidney J 7: 45-48.

6. Stegeman CA, Tervaert JW, Jong PE de, Kallenberg CG (2006) Trimethoprim-sulfamethoxazole (co-trimoxazole) for the prevention of relapses of Wegener's granulomatosis. Dutch Co-Trimoxazole Wegener Study Group. N Engl J Med 335: 16-20.

7. Reinhold-Keller E, Groot K De, Rudert H, Nölle B, Heller M, et al. (1966) Response to trimethoprim/sulfamethoxazole in Wegener's granulomatosis depends on the phase of disease. QJM 89: 15-23.

8. Zarogoulidis P, Papanas N, Kioumis I, Chatzaki E, Maltezos E, et al. (2012) Macrolides: from in vitro anti-inflammatory and immunomodulatory properties to clinical practice in respiratory diseases. Eur J Clin Pharmacol 68: 479-503.
9. Rubin BK, Henke MO (2004) Immunomodulatory activity and effectiveness of macrolides in chronic airway disease. Chest 125: 70S-78S.

10. Essilfie AT, Horvat JC, Kim RY, Mayall JR, Pinkerton JW, et al. (2015) Macrolide therapy suppresses key features of experimental steroidsensitive and steroid-insensitive asthma. Thorax 70:458-467.

11. Ratzinger F, Haslacher H, Poeppl W, Hoermann G, Kovarik JJ, et al. (2014) Azithromycin suppresses CD4+ T-cell activation by direct modulation of mTOR activity. Sci Rep 4: 7438.

12. Fouka E, Lamprianidou E, Arvanitidis K, Filidou E, Kolios G, et al. (2014) Low-Dose Clarithromycin Therapy Modulates Th17 Response In NonCystic Fibrosis Bronchiectasis Patients. Lung 192: 849-855.

13. Nogueira E, Hamour S, Sawant D, Henderson S, Mansfield N, et al. (2010) Serum IL-17 and IL-23 levels and autoantigen-specific Th17 cells are elevated in patients with ANCA-associated vasculitis. Nephrol Dial Transplant 25: 2209-2217.

14. Liu Z, Zhang H, Liu Z, Xing C, Fu P, et al. (2015) Multitarget Therapy for Induction Treatment of Lupus Nephritis: A Randomized Trial. Ann Intern Med 162: 18-26.

15. Hochegger K, Jansky GL, Soleiman A, Wolf AM, Tagwerker A, et al. (2008) Differential effects of rapamycin in anti-GBM glomerulonephritis. J Am Soc Nephrol 19: 1520-1529. 